\title{
Pharmacokinetic comparison between pelvic transarterial chemoembolization and transcatheter arterial chemotherapy in an animal model
}

\author{
TIANRONG SONG, WEI WANG, PING LIU and CHUNLIN CHEN \\ Department of Obstetrics and Gynecology, Nanfang Hospital, Southern Medical University, \\ Guangzhou 510515, Guangdong Province, P.R. China
}

Received March 26, 2009; Accepted May 25, 2009

DOI: $10.3892 / \mathrm{mmr} \_00000154$

\begin{abstract}
Transcatheter arterial chemotherapy (TAC) and transarterial chemoembolization (TACE) have greatly benefited patients suffering from gynecologic malignancies. In cervical cancer treatment, TACE appears to be more effective than TAC. We compared the pharmacokinetics of TACE with those of TAC in an animal study. Twelve female dogs were randomly divided into 2 groups $(n=6)$, and carboplatin was applied at a concentration of $1.2 \mathrm{mg} / \mathrm{kg}$. Carboplatin and lipiodol-carboplatin were administered by perfusion into the bilateral internal iliac arteries of the animals in the two groups, respectively. Plasma and tissues from the ovaries and uterus were collected for analysis at various time points after TAC or TACE. Pharmacokinetic analysis indicated that the uterine tissue area under the concentration (AUC) of carboplatin was approximately 2 times higher in the TACE group than in the TAC group $(\mathrm{P}<0.01)$. By contrast, the ovarian tissue AUC of carboplatin was much lower in the TACE group than in the TAC group $(\mathrm{P}<0.01)$, and was much lower than the uterine tissue AUC $(\mathrm{P}<0.01)$ in both groups. The plasma AUC of carboplatin was more than 2 times higher in the TACE group than in the TAC group $(\mathrm{P}<0.01)$. In conclusion, a pharmacological advantage of TACE treatment with lipiodolcarboplatin for gynecologic malignancies was observed.
\end{abstract}

\section{Introduction}

Pelvic arterial chemotherapy has been applied in gynecologic malignancies since the 1960s (1). Transcatheter arterial chemotherapy (TAC) and transarterial chemoembolization (TACE) are therapeutic modalities used for pre-surgery preparation for gynecologic malignancies. Both treatments

Correspondence to: Dr Chunlin Chen or Dr Ping Liu, Department of Obstetrics and Gynecology, Nanfang Hospital, Southern Medical University, Guangzhou 510515, Guangdong Province, P.R. China E-mail: jieru@163.com; lpivy@126.com

Key words: transcatheter arterial chemotherapy, transarterial chemoembolization, carboplatin, pharmacokinetic are capable of shrinking tumors and reducing tumor stage in order to make surgery more feasible (2), particularly in patients with large tumors. TACE and TAC also enhance radiotherapeutic effects (3).

Transcatheter arterial embolization (TAE) was initially used to occlude vascular anomalies $(4,5)$, and has recently been developed as a means of closing tumor blood vessels in order to reduce tumor size and prevent bleeding. Soga et al (6) used TAE alone to treat unresectable hepatocellular carcinoma and achieved satisfactory curative effects. Kato et al (7) reported the successful combined use of TAE and TAC in the treatment of renal cell carcinoma. Since then, many studies on TACE in hepatocellular carcinoma or renal cell carcinoma have been carried out, with the aim of imroving patient survival rates (8-10).

Randomized controlled trials have shown that TACE appears to be more effective than TAC in hepatocellular carcinoma and cervical cancer treatment $(11,12)$. These studies reported the clinical effects of tumor reduction and the patient survival rate under TACE treatment. However, they did not investigate pharmacokinetic data, such as the AUC, which serve as important assessment factors indicative of anticancer effect. This may be due to the fact that the evaluation of the pharmacological parameters of TACE and TAC is complicated by the need to collect multiple samples during surgery and the unavailability of most target organs for sampling on account of their anatomical position.

The present study was undertaken to pharmacokinetically compare the antitumor efficacies of TACE and TAC. To overcome the aforementioned obstacle, a canine model was designed to measure antitumor drug concentrations in the plasma and tissues of the ovaries and uterus.

\section{Materials and methods}

Animals. Twelve female Chinese rural dogs (average weight $15 \mathrm{~kg}$, range 13-18) were obtained from the Animal Laboratory for Research, University of Sun Yat-Sen Medical Sciences. All experiments were performed according to the Guidelines for Animal Experiments of the University of Sun Yat-Sen. The dogs were randomly divided into 2 groups: the TAC group $(n=6)$ and the TACE group $(n=6)$. 
Table I. Mean platinum concentrations $\pm \mathrm{SD}(\mu \mathrm{g} / \mathrm{g})$ in uterine tissue samples from the TAC and TACE groups at various time points after the start of infusion.

\begin{tabular}{|c|c|c|c|c|c|c|c|c|}
\hline \multirow[b]{2}{*}{ Group } & \multicolumn{8}{|c|}{ Time points (min) } \\
\hline & 0 & 5 & 15 & 30 & 45 & 60 & 120 & 240 \\
\hline TAC & $189.77 \pm 77.20$ & $150.53 \pm 26.09$ & $98.30 \pm 40.64$ & $33.96 \pm 7.92$ & $25.13 \pm 9.84$ & $5.16 \pm 6.69$ & $9.75 \pm 3.49$ & $7.66 \pm 2.80$ \\
\hline TACE & $185.26 \pm 45.22$ & $22.36 \pm 50.51$ & $149.72 \pm 67.70$ & $71.52 \pm 34.78$ & $2.35 \pm 32.06$ & $58.03 \pm 20.31$ & $55.03 \pm 29.07$ & $39.09 \pm 18.17$ \\
\hline P-value & $>0.05$ & $<0.01$ & $>0.05$ & $<0.05$ & $>0.05$ & $<0.01$ & $<0.05$ & $<0.05$ \\
\hline
\end{tabular}

Drugs. Carboplatin $\left(\mathrm{C}_{6} \mathrm{H}_{12} \mathrm{~N}_{2} \mathrm{O}_{4} \mathrm{Pt}\right)$ (Paraplatin ${ }^{\circledast}$ lyophilized powder, $100 \mathrm{mg} / \mathrm{vial}$; Bristol-Myers Squibb, Princeton, NJ, USA) was dissolved in $5 \%$ glucose solution at a stock concentration of $10 \mathrm{mg} / \mathrm{ml}$. For infusion in the TAC group, a concentration of $1.2 \mathrm{mg} / \mathrm{kg}$ body weight Carboplatin was used. This concentration is equivalent to a human dose of $300 \mathrm{mg} / \mathrm{m}^{2}$, commonly applied in clinical settings (13). Lipiodol-carboplatin was prepared by adding $0.2 \mathrm{ml} / \mathrm{kg}$ lipiodol (Lipiodol Ultra-Fluid, Xudong Haipu, Shanghai, China) to the $1.2 \mathrm{mg} / \mathrm{kg}$ carboplatin solution, then subjecting the mixture to sonication for $20 \mathrm{~min}$. The lipiodol emulsified with carboplatin-lipiodol-carboplatin was applied in the TACE group. Both carboplatin solutions were prepared immediately prior to injection.

Surgical procedure. Dogs were intravenously anesthetized with $3 \%$ nembutal sodium $(30 \mathrm{mg} / \mathrm{kg})$. After general anesthesia, a median incision was made in the lower abdominal region. The peritoneum was opened, and the bilateral internal iliac arteries were exposed. Two 4-Fr angiographic catheters (Vanguard $\mathrm{Dx}^{\circledR}$, Medrad Inc., Indianola, PA, USA) were inserted $1 \mathrm{~cm}$ below the bilateral bifurcation of the internal iliac artery via bilateral umbilical arterial puncture. Methylene blue was perfused into the bilateral internal iliac artery. Staining of both sides of the uterine body was taken to indicate successful catheterization. The carboplatin solution in the TAC group and the lipiodol-carboplatin mixture in the TACE group were respectively divided into two equal parts and infused through the catheters at a flow rate of $2.5 \mathrm{ml} / \mathrm{min}$.

Determination of tissue and plasma platinum. Plasma drug concentrations were measured at 7 time points during infusion. At each time point, $2 \mathrm{ml}$ blood was drawn from the forelimb vein. Plasma was prepared by centrifuging the blood at $1,500 \mathrm{x} \mathrm{g}$ for $30 \mathrm{~min}$. Uterine tissue samples $(200 \mathrm{mg})$ were collected at 8 time points over the same period as the plasma collection. Ovarian tissue samples $(200 \mathrm{mg})$ were collected at 6 time points following the end of infusion. The tissue sample collections were followed by a saline flush. The plasma and tissue samples were stored at $-20^{\circ} \mathrm{C}$ until measurement of drug concentrations. Plasma and tissue concentrations of carboplatin were determined by graphite-furnace Zeeman atomic absorption spectrometry $(14,15)$. Measurements were conducted at the Medical Center Laboratory of Sun Yat-Sen University.

Statistical analysis. The means \pm SD of the platinum concentrations in the plasma and tissue samples were calculated, and the independent sample t-test was used to statistically

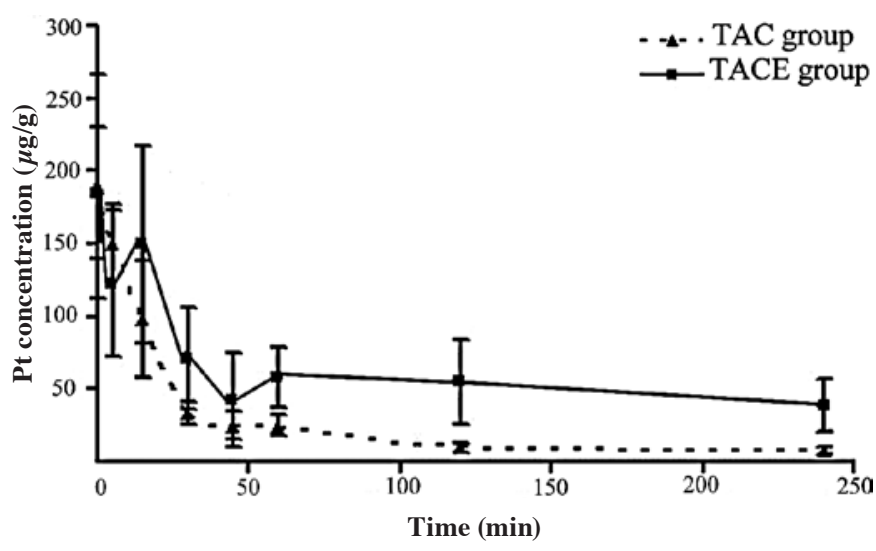

Figure 1. Mean concentration-time curves of platinum $(\mathrm{Pt})$ in the uterine tissues of the TAC and TACE groups after the start of infusion.

assess intergroup differences (SPSS II 11.0.1 J; SPSS Inc., Chicago, IL, USA). The level of significance was set at $\mathrm{P}<0.05$. AUC analysis was conducted using the non-compartment model (plasma concentration data, i.v. Bolus administration; Pharmacokinetics and Bioavailability Program Package v2.1).

\section{Results}

Platinum concentrations in uterine tissues. Table I and Fig. 1 show the time course of platinum concentrations in the uterine tissues from the TACE and TAC treatment groups. Uterine tissue platinum concentrations were significantly lower in the TAC group compared to the TACE group at 5 time points (Table I). No significant differences were observed in uterine tissue platinum concentrations between the two groups at the remaining time points. The mean uterine tissue platinum concentration was lower in the TAC group compared to the the TACE group 15 min after the end of infusion. Platinum concentrations in the uterine tissues of both groups reached peak levels immediately after the end of infusion (Fig. 1).

Platinum concentrations in ovarian tissues. Table II and Fig. 2 show the time course of platinum concentrations in the ovarian tissues from the TACE and TAC treatment groups. Ovarian tissue platinum concentrations were significantly higher in the TAC group compared to the TACE group at 30 and $60 \mathrm{~min}$ after the end of infusion $(\mathrm{P}<0.05$ and $<0.01$, respectively; Table II). No significant differences were detected in ovarian tissue platinum concentrations between the two groups at the 
Table II. Mean platinum concentrations $\pm \mathrm{SD}(\mu \mathrm{g} / \mathrm{g})$ in ovarian tissue samples from the TAC and TACE groups at various time points after the start of infusion.

Time points (min)

\begin{tabular}{lccccrr} 
& \multicolumn{1}{c}{ Group } & 0 & 60 & 120 & 180 & 240 \\
\hline TAC & $13.74 \pm 2.05$ & $19.38 \pm 6.13$ & $30.50 \pm 7.53$ & $13.52 \pm 1.73$ & $8.14 \pm 4.11$ & $5.92 \pm 2.04$ \\
TACE & $7.89 \pm 3.55$ & $4.70 \pm 1.05$ & $4.75 \pm 1.05$ & $8.60 \pm 2.92$ & $15.79 \pm 3.90$ & $4.63 \pm 1.96$ \\
P-value & $>0.05$ & $<0.05$ & $<0.01$ & $>0.05$ & $>0.05$ & $>0.05$ \\
\hline
\end{tabular}

Table III. Mean platinum concentrations $\pm \mathrm{SD}(\mu \mathrm{g} / \mathrm{ml})$ in plasma from the TAC and TACE groups at various time points after the start of infusion.

\begin{tabular}{lccccccc}
\hline & \multicolumn{7}{c}{ Time points (min) } \\
\cline { 2 - 8 } Group & 0 & 5 & 15 & 30 & 60 & 120 & 240 \\
\hline TAC & $13.96 \pm 4.12$ & $15.02 \pm 4.27$ & $10.20 \pm 2.59$ & $7.68 \pm 1.55$ & $6.60 \pm 4.01$ & $4.04 \pm 2.17$ & $1.70 \pm 0.74$ \\
TACE & $8.43 \pm 2.71$ & $7.33 \pm 1.55$ & $4.13 \pm 1.34$ & $3.93 \pm 1.94$ & $2.39 \pm 0.80$ & $1.28 \pm 0.40$ & $0.87 \pm 0.45$ \\
P-value & $>0.05$ & $<0.01$ & $<0.01$ & $<0.01$ & $>0.05$ & $<0.05$ & $<0.05$ \\
\hline
\end{tabular}

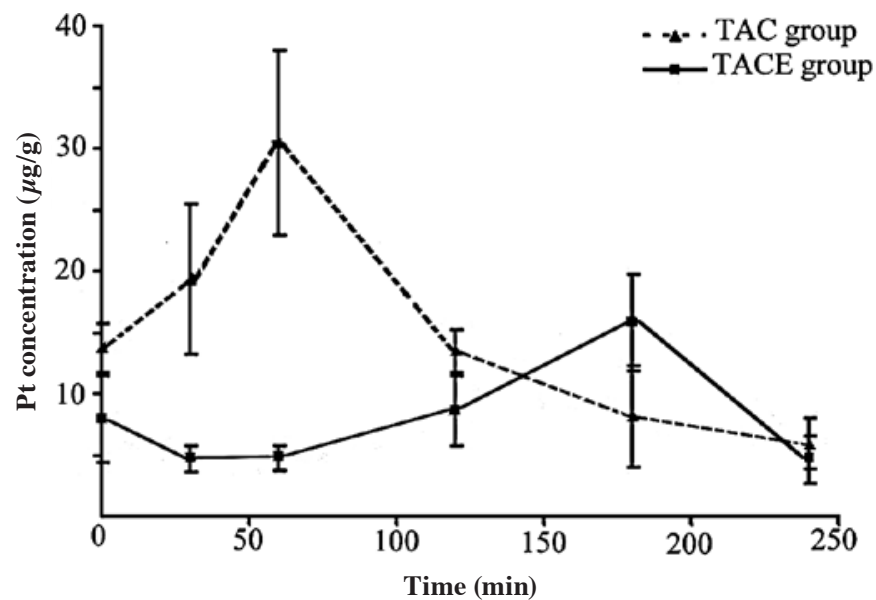

Figure 2. Mean concentration-time curves of platinum $(\mathrm{Pt})$ in the ovarian tissues of the TAC and TACE groups after the start of infusion.

remaining time points. Mean ovarian tissue platinum concentrations were higher in the TAC group than in the TACE group, except at $180 \mathrm{~min}$ after the end of infusion. Peak platinum concentration levels in the ovarian tissues were noted at 60 and $180 \mathrm{~min}$ in the TAC and TACE groups, respectively (Fig. 2).

Platinum concentrations in plasma. Sequential changes in platinum concentration are shown in Table III. Platinum concentrations were significantly higher in the TAC group compared to the TACE group, except immediately and at $60 \mathrm{~min}$ after the end of infusion. The time course of platinum concentrations in the plasma of the two groups is shown in Fig. 3. Mean platinum concentrations were higher in the TAC group than in the TACE group at each time point. Peak platinum concentration levels were observed within $5 \mathrm{~min}$ of the end of infusion in both groups.

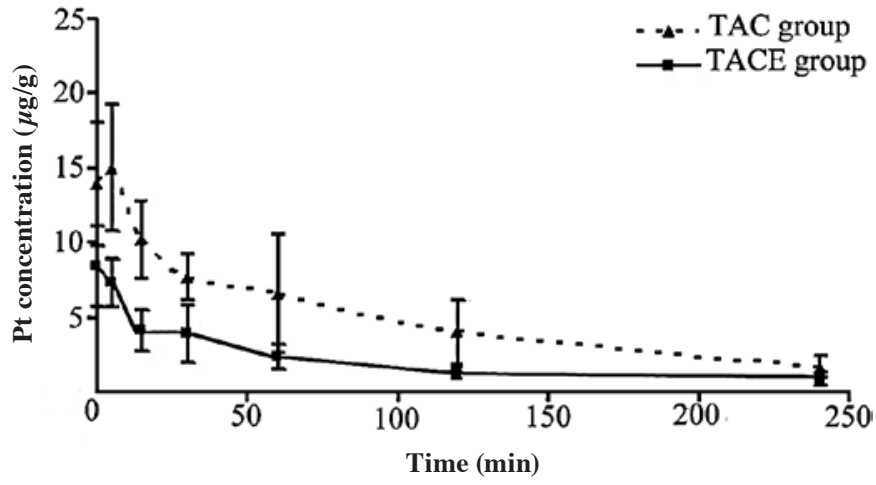

Figure 3. Mean concentration-time curves of platinum $(\mathrm{Pt})$ in the plasma of the TAC and TACE groups after the start of infusion.

Pharmacokinetic parameters. The calculated pharmacokinetic parameters are listed in Table IV. Uterine chemoembolization resulted in a significantly higher AUC in the TACE group compared to the TAC group $(\mathrm{P}<0.01)$. However, no significant difference between the two groups was detected at the peak concentration.

Comparisons between the ovarian tissues of the two groups revealed a significant increase in the AUC with TAC treatment compared to TACE treatment $(\mathrm{P}<0.01)$. In addition, the peak concentration of platinum within the ovarian tissues was significantly higher in the TAC group compared to the TACE group $(\mathrm{P}<0.01)$. The AUC of the plasma was also significantly higher in the TAC group compared to the TACE group $(\mathrm{P}<0.01)$, as was the peak concentration of platinum in the plasma (Table IV).

Comparisons between the uterine and ovarian tissues of both treatment groups revealed a significant increase in AUC 
Table IV. Pharmacokinetic parameters of the TAC and TACE groups.

\begin{tabular}{|c|c|c|c|c|c|c|}
\hline \multirow[b]{2}{*}{ Pharmacokinetic parameters } & \multicolumn{3}{|c|}{ TACE group } & \multicolumn{3}{|c|}{ TAC group } \\
\hline & Uterine tissue & Ovarian tissue & Plasma & Uterine tissue & Ovarian tissue & Plasma \\
\hline $\mathrm{T}_{\max }(\mathrm{h})$ & $0.14 \pm 0.13$ & $3.00 \pm 0$ & $0.03 \pm 0.02$ & $0.67 \pm 0.09$ & $0.92 \pm 0.2$ & $0.04 \pm 20.57$ \\
\hline $\mathrm{C}_{\max }\left(\mu \mathrm{g} / \mathrm{ml}^{-1}\right)\left(\mu \mathrm{g} / \mathrm{g}^{-1}\right)$ & $211.73 \pm 20.57$ & $16.45 \pm 4.27$ & $8.92 \pm 2.7$ & $213 \pm 43.68$ & $31.03 \pm 6.86$ & $16.73 \pm 3.92$ \\
\hline $\mathrm{t}_{1 / 2}(\mathrm{~h})$ & $8.9 \pm 0.68$ & $3.6 \pm 0.22$ & $0.66 \pm 0.29$ & $0.43 \pm 0.71$ & $0.51 \pm 0.5$ & $13.4 \pm 0.34$ \\
\hline $\operatorname{CL}\left(1 / \mathrm{h}^{-1} \times \mathrm{kg}^{-1}\right)$ & & & $0.1 \pm 0$ & & & $0.04 \pm 0.01$ \\
\hline $\mathrm{K}_{\mathrm{el}}\left(\mathrm{h}^{-1}\right)$ & $0.2 \pm 0.03$ & $1.16 \pm 0.41$ & $0.39 \pm 0.06$ & $0.43 \pm 0.12$ & $0.51 \pm 0.16$ & $0.48 \pm 0.12$ \\
\hline $\mathrm{AUC}_{0-\mathrm{t}}\left(\mu \mathrm{g} / \mathrm{ml}^{-1} \mathrm{~h}\right)\left(\mu \mathrm{g} / \mathrm{g}^{-1} / \mathrm{h}\right)$ & $239.55 \pm 69.78$ & $36.08 \pm 6.43$ & $8.62 \pm 1.6$ & $98.73 \pm 8.93$ & $60.43 \pm 7.8$ & $20.05 \pm 6.55$ \\
\hline $\operatorname{AUC}_{0-\infty}\left(\mu \mathrm{g} / \mathrm{ml}^{-1} / \mathrm{h}\right)\left(\mu \mathrm{g} / \mathrm{g}^{-1} / \mathrm{h}\right)$ & $295.92 \pm 80.53$ & $40.7 \pm 7.27$ & $16.43 \pm 2.39$ & $112.08 \pm 10.93$ & $70.83 \pm 10.96$ & $29.08 \pm 6.48$ \\
\hline MRT (h) & $1.57 \pm 0.19$ & $2.3 \pm 0.12$ & $1.3 \pm 0.15$ & $0.93 \pm 0.08$ & $1.5 \pm 0.13$ & $1.29 \pm 0.10$ \\
\hline $\mathrm{Vd}\left(1 \times \mathrm{kg}^{-1}\right)$ & & & $0.22 \pm 0$ & & & $0.095 \pm 0.03$ \\
\hline
\end{tabular}

$\mathrm{T}_{\max }$, time of maximal plasma concentration; $\mathrm{C}_{\max }$, peak concentration of plasma or tissue; $\mathrm{t}_{1 / 2}$, half-life of the elimination phase; CL, total body clearance; AUC, area under the plasma or tissue concentration-time curve; $\mathrm{AUC}_{0-\mathrm{t}}, \mathrm{AUC}$ to $240 \mathrm{~min}$; $\mathrm{AUC} \mathrm{C}_{0-\infty}, \mathrm{AUC}$ to infinite time; MRT, mean residence time; $\mathrm{Vd}$, apparent volume of distribution; $\mathrm{K}_{\mathrm{el}}$, elimination rate constant.

in the uterine tissue $(\mathrm{P}<0.01)$. The peak concentration of platinum was significantly higher in the uterine tissue compared to the ovarian tissue $(\mathrm{P}<0.01)$.

\section{Discussion}

In recent years, chemotherapy has been widely accepted as a beneficial treatment modality for cervical cancer $(16,17)$. However, most conventional anticancer chemotherapeutic agents have a major limitation, their lack of tumor selectivity, which requires increasing doses of an antitumor drug to be administered for the desired therapeutic effect to be acheived. However, with increases in plasma concentration levels of an antitumor drug, an increase in toxic side effects is observed. Reducing drug concentration levels in the plasma is an efficient way to alleviate these negative effects.

The present study demonstrated that the peak level of carboplatin in plasma was much lower in the TACE group than in the TAC group, and that the plasma AUC of carboplatin was more than 2 times higher in the TAC group than in the TACE group. These results indicate that systemic drug concentrations were lower with TACE treatment, which suggests that TACE would have fewer side effects than TAC.

Although several efficacy studies on radiological interventional treatment for gynecologic malignancies have been conducted $(18,19)$, standard interventional therapy has yet to be established. The current interventional treatment in gynecologic malignancies relies on therapeutic experience in the treatment of hepatic carcinoma. TAE and TAC, used alone or in combination in hepatic carcinoma, have been reported on $(20,6)$. Comparison studies of TAE and TAC have shown that TAE treatment is associated with a higher survival rate than TAC in unresectable hepatic carcinoma patients $(20,6)$. On the other hand, in a randomized prospective trial conducted to evaluate the efficacy of epirubicin alone vesus lipiodol emulsion containing the same dose of epirubicin in intrahepatic arterial infusion chemotherapy for hepatic carcinoma patients, lipiodol emulsion with epirubicin was shown to be more effective than epirubicin alone (11). The results of studies on the treatment of locally advanced cervical cancer were consistent with those on hepatic carcinoma: the tumor reduction ratio was significantly higher for the combination of intra-arterial cisplatin and gelfoam compared to gelfoam used alone, and cisplatin combined with other anticancer agents infused via the arteries had similar efficacy (12).

The AUC represents the total dose absorbed by tissue after one administration at a specific dose and time point. It is important to determine the bioavailability of a drug and to compare its efficacy with different administration routes. Since the AUC of tumor tissue is more indicative of drug absorption than the AUC of plasma, we compared the AUC of tumor tissues under two administration routes, TACE and TAC. Our results show that the uterine tissue AUC of carboplatin in the TACE group was approximately 2 times higher than that of the TAC group. Compared to TAC, TACE resulted in a significantly higher AUC of carboplatin without reducing the peak level of carboplatin in the uterine tissue. This indicates that TACE is more efficient than TAC.

In this study, carboplatin was infused via the internal iliac arteries to the uterus. However, carboplatin was washed out rapidly during infusion, and its retention was temporal for more absorption by the uterine tissues (21). The use of lipiodol as an embolic agent blocked the arterioles, thus the velocity of the flow in the uterus was reduced. As a result, the anticancer drug slowly flowed back into systemic circulation. The emulsion of lipiodol and carboplatin allowed retention inside the tumor for a long period of time, enhancing local efficacy. Notably, we found that the uterine tissue AUC of carboplatin was much higher in the TACE group than in the TAC group.

Ovarian failure and infertility are side effects associated with chemotherapy in young cancer patients. The mechanisms of these negative effects are not completely understood. However, it is evident that ovarian damage following chemotherapy is both drug and dose dependent (22). Cisplatin 
administered for gynecologic malignancies results in ovarian failure with an odds ratio of 1.77 (22). Carboplatin, a second generation platinum drug, exhibits non-specific effects on normal cell function. These unwanted side effects are doserelated; thus, a lower drug concentration should result in less ovarian injury. Our study demonstrated that the ovarian tissue AUC of carboplatin was much lower in the TACE group than in the TAC group, that the peak level of carboplatin in the ovaries was approximately 2 times higher in the TAC group than in the TACE group, and that the AUC of ovarian tissue was much lower than that of uterine tissue in both groups. The animal experiments conducted indicate that TACE, as a neoadjuvant chemotherapy, may better preserve ovarian function in patients with locally advanced cervical carcinomas without ovarian invasion.

Furtherclinical studies are, however, needed. Hemodynamic analysis of carboplatin infused via the internal iliac artery to the uterine and ovarian tissues showed that most of the drug was delivered to the uterus rather than to the ovaries, as the uterus receives a more abundant blood supply. This may account for the higher AUC of carboplatin observed in the uterus of both groups compared to the ovaries. Additionally, the mean diameters of the ovarian branch of the uterine artery and the uterine artery itself were less than $1.5 \mathrm{~mm}$, meaning the 4-Fr catheter, which has an external diameter of $1.33 \mathrm{~mm}$, could only be inserted into the internal iliac artery. Carboplatin was thus by necessity infused into the ovaries via the ovarian branch of the uterine artery. As the lipiodol-carboplatin administered in the TACE group is more viscous than carboplatin alone, it had more difficulty flowing into such small-diameter vessels as the ovarian branches of the uterine artery. This may also explain why, in the TACE group, a reduced amount of the drug reached the ovarian tissues.

In conclusion, we observed the pharmacological advantages of TACE for carboplatin delivery in the treatment of gynecologic malignancies. TACE is likely to have a stronger antitumor effect and reduced systemic side effects compared to TAC. Pharmacological analysis may aid in establishing standard interventional treatment for gynecologic malignancies.

\section{Acknowledgements}

We thank Rangke Wu of the Institute of Foreign Language, Southern Medical University, China, and Changping Zou of the Department of Obstetrics and Gynecology, University of Connecticut, USA for revising the manuscript. Support for the study was provided by the Natural Science Foundation of Guangdong Province (no. 06024389).

\section{References}

1. Cromer JK, Bateman JC, Berry GN, Kennelly JM, Klopp CT and Platt LI: Use of intra-arterial nitrogen mustard therapy in the treatment of cervical and vaginal cancer. Am J Obstet Gynecol 63: 538-548, 1952.

2. Fujiwara K: Antitumor effects and clinical problems of transcatheter intraarterial infusion for uterine cervical cancer. Gan To Kagaku Ryoho 33: 1236-1240, 2006.

3. Motoyama S, Hamana S, Ku Y, Laoag-Fernandez JB, Deguchi M, Yoshida S, Tominaga M, Iwasaki T, Ohara N and Maruo T: Neoadjuvant high-dose intraarterial infusion chemotherapy under percutaneous pelvic perfusion with extracorporeal chemofiltration in patients with stages iiia-iva cervical cancer. Gynecol Oncol 95: $576-582,2004$.
4. Newton TH and Adams JE: Angiographic demonstration and nonsurgical embolization of spinal cord angioma. Radiology 91: 873-876 passim, 1968.

5. Sano K: Intracranial arterio-venous malformation with special reference to its treatment. No To Shinkei 17: 360-364, 1965.

6. Soga K, Nomoto M, Ichida T, Aoyagi Y, Ozaki T and Ichida F: Clinical evaluation of transcatheter arterial embolization and one-shot chemotherapy in hepatocellular carcinoma. Hepatogastroenterology 35: 116-120, 1988.

7. Kato T, Nemoto R, Mori H, Takahashi M and Tamakawa Y: Transcatheter arterial chemoembolization of renal cell carcinoma with microencapsulated mitomycin c. J Urol 125: 19-24, 1981.

8. Yang CF, Ho YZ, Chang JM, Chiang RH, Lai KH, Lee SD, Tsai YT, Lui WY, Liu TJ and Chen GH: Transcatheter arterial chemoembolization for hepatocellular carcinoma. Cancer Chemother Pharmacol 23: S26-S28, 1989.

9. Timm S, Sotiropoulos GC, Draier M, Knüpffer J, Steger U and Lang H: Selective transarterial chemoembolization of advanced hepatocellular carcinomas: a reasonable palliative option. Transplant Proc 40: 3179-3181, 2008.

10. Vaicekavicius E and Pranculis A: Transcatheter renal arterial embolization in malignant renal neoplasms: clinical results and indications for use of the method in multi-profile hospitals. Medicina (Kaunas) 38: 888-891, 2002.

11. Yoshikawa M, Saisho H, Ebara M, et al: A randomized trial of intrahepatic arterial infusion of 4'-epidoxorubicin with lipiodol versus 4 '-epidoxorubicin alone in the treatment of hepatocellular carcinoma. Cancer Chemother Pharmacol 33: S149-S152, 1994.

12. Ikeda O, Mizukami N, Murata Y, Arakawa A, Katabuchi H, Okamoto H, Yasunaga T, Tsunawaki A and Yamashita Y: Randomized comparison of intra-arterial chemotherapy versus intra-arterial chemotherapy and gelfoam embolization for treatment of advanced cervical carcinoma. Cardiovasc Intervent Radiol 28: 736-743, 2005.

13. Sun RY: Quantitative Pharmacology (in Chinese). People's Health Publishing House, Beijing, pp245-246, 1989.

14. Meerum-Terwogt JM, Tibben MM, Welbank H, Schellens JH and Beijnen JH: Validated method for the determination of platinum from a liposomal source (SPI-77) in human plasma using graphite furnace Zeeman atomic absorption spectrometry. Fresenius J Anal Chem 366: 298-302, 2000.

15. Tibben MM, Rademaker-Lakhai JM, Rice JR, Stewart DR, Schellens JH and Beijnen JH: Determination of total platinum in plasma and plasma ultrafiltrate, from subjects dosed with the platinum-containing $\mathrm{N}$-(2-hydroxypropyl)methacrylamide copolymer AP5280, by use of graphite-furnace Zeeman atomicabsorption spectrometry. Anal Bioanal Chem 373: 233-236, 2002.

16. Landoni F, Parma G, Peiretti M, Zanagnolo V, Sideri M, Colombo $\mathrm{N}$ and Maggioni A: Chemo-conization in early cervical cancer. Gynecol Oncol 107: S125-S126, 2007.

17. Loizzi V, Cormio G, Vicino M and Selvaggi L: Neoadjuvant chemotherapy: an alternative option of treatment for locally advanced cervical cancer. Gynecol Obstet Invest 65: 96-103, 2008.

18. Adachi S, Ogasawara T, Tsubamoto H, Oku H, Hori Y, Tsuji Y, Takemura $\mathrm{T}$ and Koyama $\mathrm{K}$ : Intravenous nedaplatin and intraarterial cisplatin with transcatheter arterial embolization for patients with locally advanced uterine cervical cancer. Int J Clin Pharmacol Res 21: 105-110, 2001.

19. Umeki H, Yamaguchi Y, Tsugata M, Wakana K, Somekawa Y, Shimabukuro K, Ohara M, Terauchi T, Ichimura M, Otsuka I and Aso T: Neoadjuvant intraarterial chemotherapy with nedaplatin, peplomycin and mitomycin c for advanced cervical cancer. Gan To Kagaku Ryoho 30: 377-382, 2003.

20. Tanikawa K, Hirai K, Kawazoe Y, Yamashita K, Kumagai M and Abe M: One-shot therapy and transcatheter arterial embolization (TAE) therapy of unresectable hepatocellular carcinoma. Gan To Kagaku Ryoho 12: 1930-1937, 1985.

21. Kurzidem M, Seidensticker P and Rassweiler J: Renal chemoembolization with mitomycin c/Ethibloc: pharmacokinetics and efficacy in an animal model. J Endourol 16: 515-518, 2002.

22. Meirow D and Nugent D: The effects of radiotherapy and chemotherapy on female reproduction. Hum Reprod Update 7: 535-543, 2001. 